\title{
Reduced expression of indoleamine 2,3-dioxygenase participates in pathogenesis of preeclampsia via regulatory $\mathbf{T}$ cells
}

\author{
XIAOQIAN LIU ${ }^{1}$, YANXIA LIU ${ }^{1}$, MEI DING ${ }^{1}$ and XIN WANG ${ }^{1,2}$ \\ ${ }^{1}$ Department of Hematology, Provincial Hospital Affiliated to Shandong University; \\ ${ }^{2}$ Department of Dignostics, Shandong Univeristy School of Medicine, Shandong, 250012, P.R.China
}

Received August 13,2010; Accepted October 22, 2010

DOI: $10.3892 / \mathrm{mmr} .2010 .395$

\begin{abstract}
Preeclampsia is a complex multi-organ disease and the leading cause of maternofetal morbidity and mortality. Low levels of indoleamine 2,3-dioxygenase (IDO) and diminished numbers of regulatory $\mathrm{T}$ cells (Tregs) have been separately reported to participate in the pathogenesis of preeclampsia. In the present study, we aimed to determine whether alterations in the expression of IDO, forkhead box P3 (Foxp3) and interleukin-18 (IL-18) contribute to the pathogenesis of preeclampsia, by measuring their levels in preeclamptic placentae and in placentae from healthy pregnant women as a control. IDO protein levels were determined by immunohistochemistry, while the mRNA levels of IDO, FoxP3 and IL-18 were measured by quantitative RT-PCR. The intensity of IDO immunostaining was lower in the preeclamptic placentae than in the normal controls, and was related to the clinical severity of the preeclampsia. Quantitative RT-PCR revealed that the IDO and IL-18 mRNA levels were reduced in a correlated manner the preeclamptic placentae compared to the controls, and that the levels of FoxP3 mRNA were consistently decreased in the preeclamptic group. A positive correlation betwen FoxP3 and IDO mRNA was found. The results of the study indicate that IL-18, IDO and FoxP3 mRNA levels were decreased in the preeclamptic placentae. Reduced IDO levels in pre eclampsia may be partly influenced by IL-18, and result in the maladaptation of maternal tolerance and the pathogenesis of preeclampsia by directly reducing Tregs.
\end{abstract}

\section{Introduction}

Preeclampsia is a complex multi-organ disease and the leading cause of maternofetal morbidity and mortality $(1,2)$.

Correspondence to: Dr. Xin Wang, Department of Hemotology, Provicial Hospital Affiliated to Shandong University, 324 Jingwu Road, Jinan, Shandong, 250021, P.R. China

E-mail: xinwang55@yahoo.com.cn

Key words: preeclampsia, regulatory $\mathrm{T}$ cells, indoleamine 2,3-dioxygenase, forkhead box P3, interleukin-18
This obstetrical condition is also associated with significant fetal growth restriction, perinatal asphyxia and pre-term delivery. Although it is a common disease, its pathogenesis has yet to be fully elucidated. Increasing evidence now indicates that this disorder may have an immunological cause, in the form of allogeneic fetal rejection (3-5). In recent years, the down-regulation of indoleamine 2,3-dioxygenase (IDO) and diminished regulatory $\mathrm{T}$ cells (Tregs) in preeclampsia have been separately reported to play a crucial role in the maladaptation of maternal tolerance and the pathogenesis of preeclampsia (5-8).

As the key enzyme in tryptophan metabolism, IDO catalyzes the initial rate-limiting step of tryptophan degradation along the kynurenine pathway. In humans, IDO expression has been observed by immunohistochemistry in placentae $(9,10)$, tumor-draining lymph nodes (11) and primary tumors $(12,13)$, which in turn are infiltrated by $\mathrm{CD} 4{ }^{+} \mathrm{CD} 25^{+}$Treg cells. The close relationship between IDO activity and Treg cells has recently been the focus of intense study, since this positive correlation has been found to play a critical role in the pathogenesis of tumors and autoimmune diseases, as well as in spontaneous abortion. Thus, we hypothesized that both IDO expression levels and the number of Treg cells would be reduced in placentae from preeclamptic women, and that the decrease in Treg cells would be related to the down-regulation of IDO.

Interleukin-18 (IL-18), originally described as interferon- $\gamma$ (IFN- $\gamma$ )-inducing factor, is known to induce tumor necrosis factor- $\alpha$ (TNF- $\alpha$ ) and IFN- $\gamma$ production (14). IL-18 plays an important role in the Th1-mediated immune response in conjunction with interleukin-12 (IL-12), and also stimulates the Th2 immune response in the absence of IL-12 (15). To date, IL-18 levels in preeclampsia have been measured in only a few studies, which reported contradictory results in the sera $(16,17)$ and plasma $(18,19)$ of normotensive and pre eclamptic women. Notably, not only IFN- $\gamma$, which is induced by IL-18, but also IL-18 itself proved to be independently capable of inducing IDO expression (20). In the present study, immunohistochemistry and quantitative RT-PCR were used to measure the expression levels of IDO, forkhead box P3 (Foxp3) and interleukin-18 (IL-18) in preeclamptic placentae, with the aim of determining whether alterations in their levels are related and contribute to the pathogenesis of preeclampsia. 


\section{Materials and methods}

Samples. Fresh placentae samples were collected from women with a singleton pregnancy complicated by preeclampsia $(n=16)$ and from women with a normal healthy singleton pregnancy $(\mathrm{n}=18)$. Preeclampsia was diagnosed according to the criteria of the International Society for the Study of Hypertension in Pregnancy (21). All placenta samples were obtained with informed consent according to the Declaration of Helsinki. Approval was obtained from the Ethical Committee of the Provincial Hospital affiliated to Shandong University.

Immunohistochemistry. The placentae were cut into small blocks, washed several times with phosphate buffered saline to remove excess blood, fixed for 12-16 $\mathrm{h}$ in neutral buffered formalin, and embedded in paraffin. Sections $(5 \mu \mathrm{m})$ were cut from the paraffin-embedded tissue blocks and placed onto APES-coated slides.

Immunohistochemical analyses were performed using the avidin-biotin peroxidase complex method according to standard manual methods. Sections were stained with antibodies directed against human IDO (Abcam, Cambridge, UK). Heat retrieval was performed before the addition of the primary antibody. Appropriate positive and negative controls were used for all immunohistochemical stains. Images were captured on a Leica DM 4000B microscope (Solms, Germany).

Immunostaining for IDO was scored by two independent observers without prior knowledge of the group from which the sections came. Ten fields per subject were semiquantitatively scored in each section as follows: 0 , no staining; 1 , weak staining; 2 , moderate staining; and 3 , strong staining (7).

Quantification of IL-18, IDO and Foxp3 mRNA. Total RNA was rapidly isolated using the RNA isolation kit (Invitrogen, Calsbad, USA). Reverse transcription was carried out with $0.5 \mu \mathrm{g}$ of the total RNA to transcribe the cDNA. Quantitative PCR was performed on an ABI 7500 Fast Instrument (Perkin Elmer, USA) using SYBR Premix Ex Taq (Takara, Dalian, China). The primers used were: human INDO, forward 5'-CCCTTCAAGTGTTTCACCAAATC-3', reverse 5'-ATG TCCTCCACCAGCAGTC-3'; human FoxP3, forward 5'-GAC TTGGTGCTGACGAACTG-3', reverse 5'-GCCTGAACAAG AGCCTTTATCC-3'; human IL-18, forward 5'-TGCATCAAC TTTGTGGCAAT-3', reverse 5'-ATAGAGGCCGATTTCCTT GG-3'; and human $\beta$-actin, forward 5'-TGGCACCCAGCA CAATGAA-3', reverse 5'-CTAAGTCATAGTCCGCCTAGA AGCA-3'. A relative quantitative assay was adopted in which the $\mathrm{Ct}$ value equalled the number of PCR cycles required for the fluorescence signal to exceed the detection threshold value. The difference between the $\mathrm{Ct}$ values of the target gene and the $\beta$-actin housekeeping gene was used to calculate the $\Delta \mathrm{Ct}$. All experiments were run in triplicate with the same thermalcycling parameters. Non-template controls and dissociation curves were used to detect primer dimer conformation and non-specific amplification. Human $\beta$-actin mRNA was used for normalization to ensure equal amounts of starting RNA.

Statistical analysis. Statistical analyses were performed using SPSS software version 16.0. Clinical data were
Table I. Study subject characteristics.

\begin{tabular}{lcc}
\hline & $\begin{array}{c}\text { Control } \\
(\mathrm{n}=18)\end{array}$ & $\begin{array}{c}\text { Preeclampsia } \\
(\mathrm{n}=16)\end{array}$ \\
\hline $\begin{array}{l}\text { Maternal age } \\
\text { (years) }\end{array}$ & $27.60 \pm 3.80$ & $31.8 \pm 4.5$ \\
$\begin{array}{l}\text { Gestational age } \\
\text { (weeks) }\end{array}$ & $38.20 \pm 1.10$ & $35.7 \pm 2.0^{\mathrm{a}}$ \\
$\begin{array}{l}\text { Mean systolic } \\
\text { pressure (mmHg) }\end{array}$ & $108.0 \pm 13.60$ & $179.8 \pm 28.0^{\mathrm{b}}$ \\
$\begin{array}{l}\text { Mean diastolic } \\
\text { pressure (mmHg) }\end{array}$ & $81.00 \pm 16.60$ & $116.2 \pm 18.1^{\mathrm{a}}$ \\
$\begin{array}{l}\text { Proteinuria } \\
\begin{array}{l}\text { Birth weight } \\
\text { of fetus }(\mathrm{g})\end{array}\end{array}$ & - & $+-++++^{\mathrm{b}}$ \\
\hline
\end{tabular}

${ }^{\mathrm{a}} \mathrm{P}<0.05,{ }^{\mathrm{b}} \mathrm{P}<0.01$ compared to the control.

normally distributed and expressed as the means \pm standard deviation (SD), and IDO, FoxP3 and IL-18 expression levels were expressed as quartiles. Statistical differences between groups were compared using the Student's $t$ test or the Mann-Whitney U test, and correlations were calculated using Spearman's Rho test. P-values $<0.05$ were considered statistically significant.

\section{Results}

The median age of the healthy and preeclamptic women was 27.6 and 31.8 years, respectively. After statistical analysis, the fetal birthweight was significantly lower and the gestational age was markedly shorter in the preeclamptic group. Preeclamptic women had elevated proteinuria and much higher blood pressure. Relevant clinical details of the subjects are presented in Table 1.

As shown in Fig. 1, immunostaining for IDO was observed almost entirely in endothelial cells and was evident in most capillaries and specific villi arteries. In the endothelial cells, IDO-positive immunostaining was distributed in both the nuclei and cytoplasm. Weak but substantial signals were also detectable in syncytiotrophoblasts, while no staining was observed in interstitial cells. No positive staining was observed in the negative control sections. Marked differences in the intensity of IDO immunostaining were observable by direct visual comparison of normal and preeclamptic placentae (Fig. 1), and were confirmed by statistical analysis of the semi-quantitative scoring $(\mathrm{P}=0.001$, Mann-Whitney $\mathrm{U}$ test) (Fig. 2).

By comparing the clinical data from the preeclamptic samples grouped according to the semi-quantitative score, the women with samples showing weak or no IDO staining were found to have significantly higher maternal blood pressure as well as a lower fetal birthweight and shorter gestational age (Table 1) than the women with moderate or strong IDO staining. 

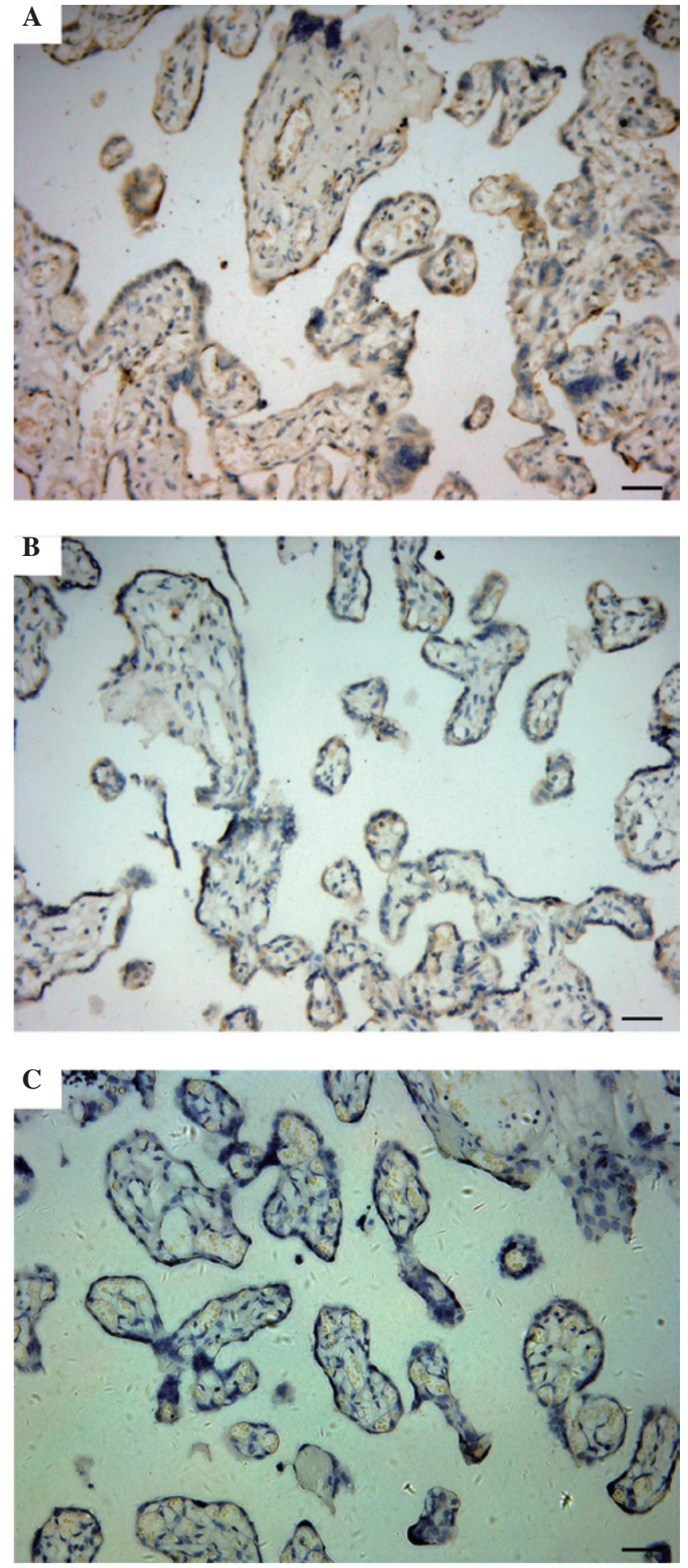

Figure 1. Immunostaining for IDO. (A) In normal placentae, IDO was expressed almost entirely in endothelial cells and was evident in most capillaries and some villi arteries. (B) Weaker signals were detected in preeclampsia placentae. (C) No staining was observed in the negative control sections. Scale bar, $40 \mu \mathrm{m}$.

In quantitative RT-PCR assays, the levels of IL-18, IDO and FoxP3 mRNA were simultaneously investigated in placentae from preeclamptic women $(n=16)$ and healthy pregnant women $(n=18)$ as controls. As shown in Fig. 3, the expression of IDO and IL-18 mRNA decreased in a correlated manner in the preeclamptic placentae compared to the controls (IL-18,

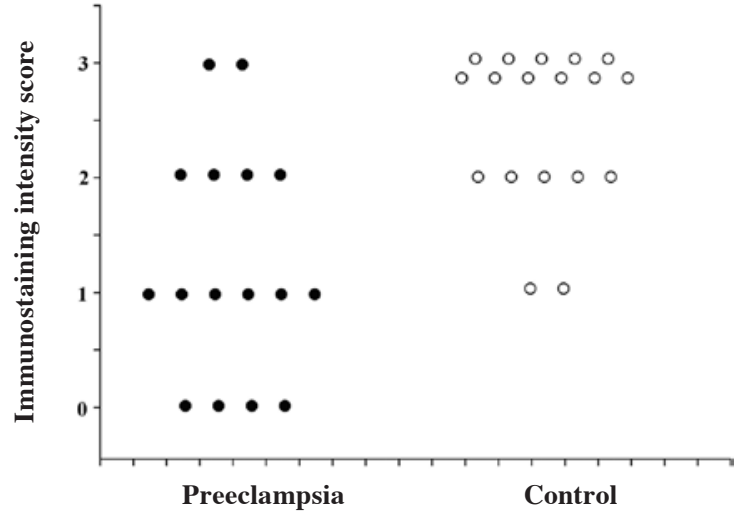

Figure 2. Semi-quantitative scoring of the results of IDO immunostaining: 0 , no staining; 1 , weak staining; 2 , moderate staining; and 3 , strong staining. Scores were significantly lower in the preeclamptic placentae than in the normal placentae.

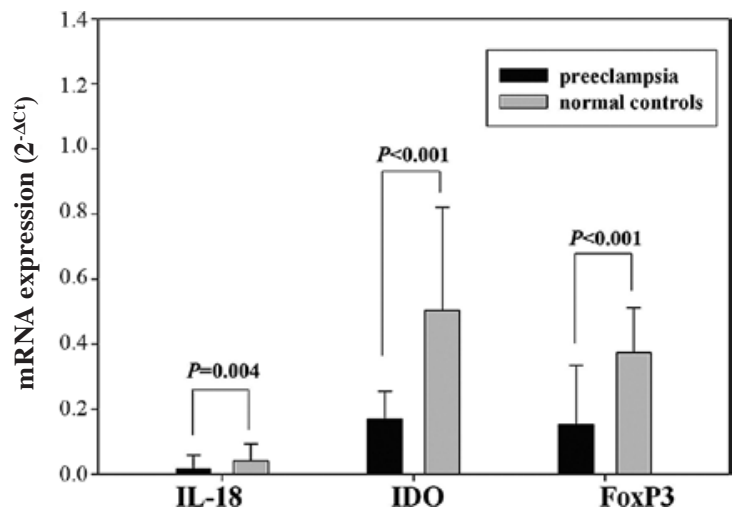

Figure 3. IL-18, IDO and FoxP3 expression determined by quantitative PCR. Levels of IL-18 and IDO mRNA were decreased in the placentae of preeclamptic patients $(n=16)$ compared to those of healthy pregnant women $(n=18)$. FoxP3 mRNA levels $(n=16)$ were significantly lower in preeclamptic placentae compared to the controls $(n=18)$.

$\mathrm{P}=0.004$; IDO, $\mathrm{P}<0.001)$. There was also a decrease in the FoxP3 mRNA level in preeclamptic women $(\mathrm{P}<0.001)$.

No significant correlation between IL-18 and IDO levels was found upon analysis of the placentae from preeclamptic women ( $\mathrm{r}=0.303, \mathrm{P}=0.254$ ) (Fig. 4A). However, the expression of IL-18 mRNA was significantly downregulated in preeclampsia samples with weak or no IDO immunostaining compared to those with moderate or strong staining $(\mathrm{P}=0.001)$ (Fig. 4B). Furthermore, in the preeclamptic placentae, a positive correlation was found between the levels of FoxP3 and IDO mRNA ( $\mathrm{r}=0.535, \mathrm{P}=0.033$ ) (Fig. 5A). To further confirm this relationship, FoxP3 mRNA levels were compared among the groups with different intensities of IDO immunostaining. The data revealed that the expression of FoxP3 mRNA was downregulated in samples with weak or no IDO staining $(\mathrm{P}=0.001)$ (Fig. 5B).

\section{Discussion}

The results of this study indicate that the levels of IL-18, IDO and FoxP3 mRNA were reduced in placentae from preeclamptic women compared to the normal controls. A 
A

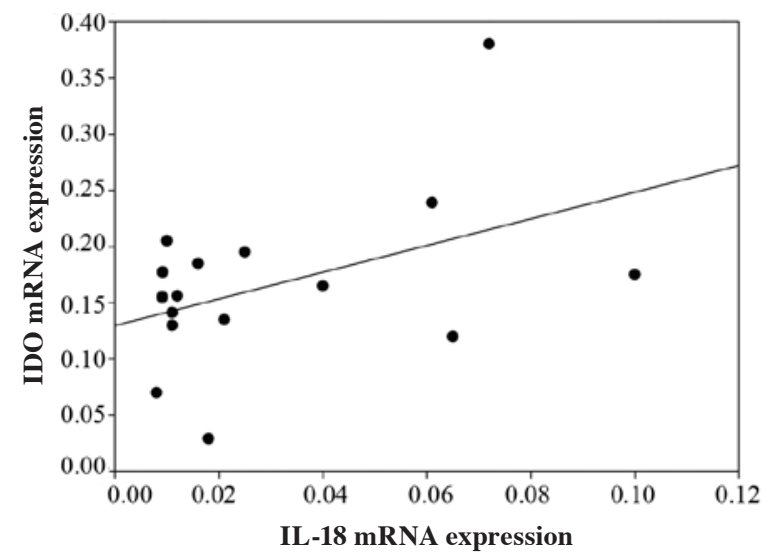

B

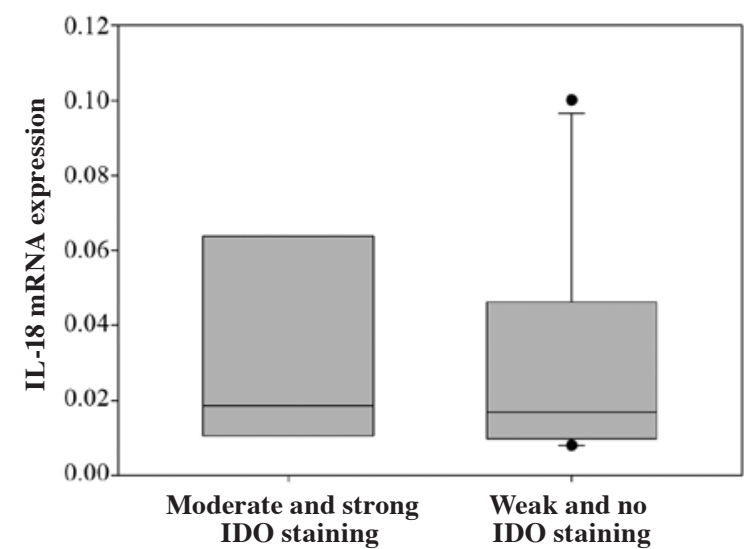

Figure 4. Correlation between the expression of IL-18 and IDO. (A) In the preeclamptic placentae, no significant correlation was found between IL-18 and IDO mRNA levels ( $\mathrm{r}=0.303, \mathrm{P}=0.254)$. (B) IL-18 mRNA level in terms of IDO immunostaining intensity. The expression of IL-18 mRNA was downregulated in the samples with weak or no IDO staining compared to those with moderate or strong staining $(\mathrm{P}=0.001)$.

\section{A}

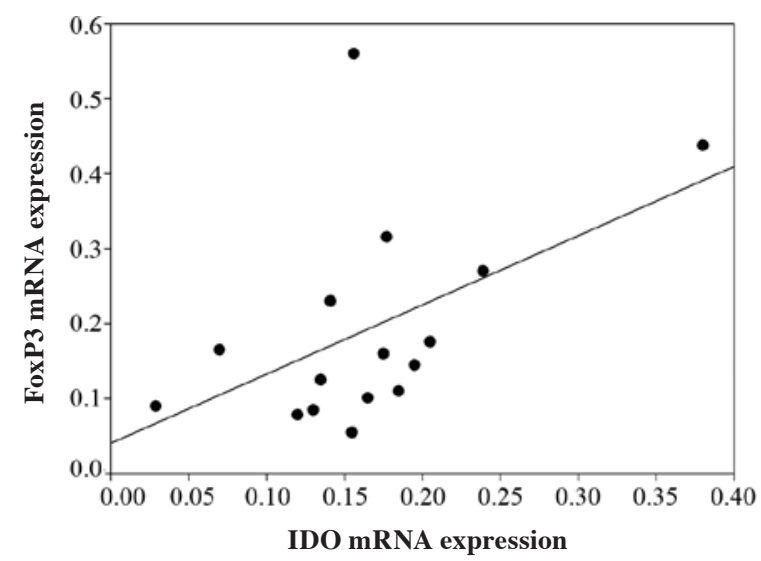

B

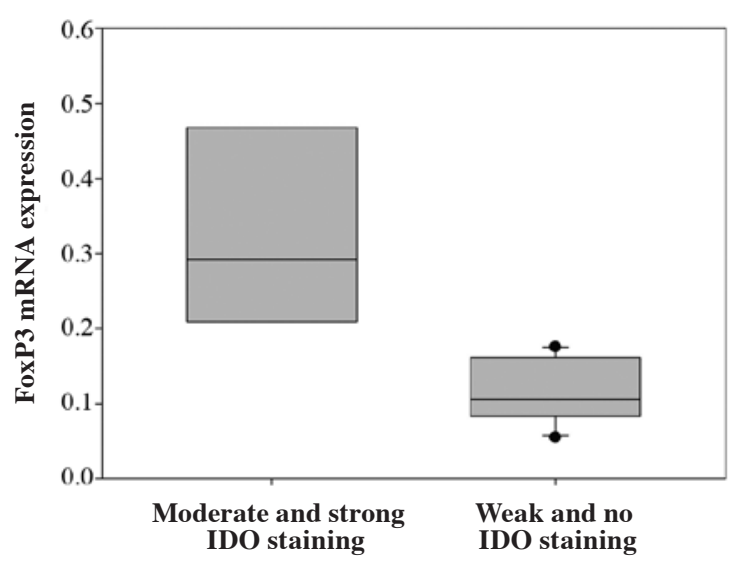

Figure 5. Correlation between the expression of FoxP3 and IDO. (A) In the preeclamptic placentae, there was a positive correlation between FOXP3 and IDO mRNA ( $\mathrm{r}=0.501, \mathrm{P}=0.003$ ). (B) FoxP3 mRNA level in terms of IDO immunostaining intensity. The expression of FoxP3 mRNA was downregulated in the samples with weak or no IDO staining compared to those with moderate or strong staining $(\mathrm{P}=0.003)$.

significantly positive correlation was found between the severity of preeclampsia and the degree of IDO staining. Furthermore, the level of FoxP3 expression was consistent with that of IDO, indicating a close relationship between IDO and Tregs. We performed a comparative study of normal and preeclamptic pregnancies. The data revealed that IL-18, IDO and FoxP3 levels were significantly down-regulated in preeclamptic placentae compared to the controls, as evidenced by IDO immunostaining and quantitative RT-PCR.

The function of IL-18 has been described in numerous inflammatory diseases, including inflammatory arthritis, insulin-dependent diabetes mellitus and pulmonary diseases (14). IL-18 plays an important role in Th1-mediated immunity in conjunction with IL-12, and also stimulates Th2 immunity in the absence of IL-12 (15). However, only a few studies have associated IL-18 to preeclampsia. Two studies have described a significant elevation of serum and/or placenta IL-18 levels in preeclampsia $(16,17)$. Others have examined the level of IL-18 in preeclamptic plasma $(18,19)$. Among these studies, Adams et al (18) did not observe any changes in the plasma IL-18 levels of normotensive and preeclamptic women, while Roland et al (19) found that IL-18 concentrations were significantly lower in the preeclamptic samples than in the controls. It has been reported that, besides preeclampsia, the early phase of gestation $(<34$ weeks) $(23,24)$ and treatment with $\beta$-methasone (18) may be related to the level of IL-18 in maternal blood. However, since previous studies found no association between IL-18 levels in plasma and gestational age (18) or $\beta$-methasone (19), we believe that the reduced IL-18 levels observed in preeclampsia are not the result of $\beta$-methasone treatment, but rather indicate a different role for IL-18 in the pathology of preeclampsia.

IL-18, originally described as IFN- $\gamma$-inducing factor, is known to induce the production of TNF- $\alpha$ and IFN- $\gamma$ (14), the latter of which has a major influence on IDO expression (25). IL-18 has also been demonstrated to induce IDO in human osteosarcoma cells, independently of IFN- $\gamma$ (20). In the present study, IL-18 and IDO mRNA levels were both downregulated in preeclampsia samples compared to the normal controls. Although no significant correlation was found between these results in the preeclampsia samples, when IL-18 levels were compared between groups with different intensities of IDO immunostaining, the level of IL-18 was significantly lower in the samples with weak or no IDO immunostaining. 
The present results regarding IDO expression are consistent with previous reports describing a relationship between IDO and the pathogenesis of preeclampsia (6). In agreement with this study, Nishizawa et al (5) demonstrated that placental IDO activity values had a significant inverse correlation with both systolic and diastolic blood pressure, indicating that the severity of preeclampsia is inversely correlated with IDO activity. The mechanism of IDO-mediated immunosuppression was initially believed to depend on the inhibition of T-cell effectors by toxic metabolites of tryptophan (26). Activated T-cells undergo cell-cycle arrest when deprived of tryptophan, thus becoming more susceptible to apoptosis (27). The importance of IDO to maternal-fetal tolerance was confirmed by demonstrating that the administration of IDO inhibitor led to higher blood pressure and proteinuria, in addition to local circulation impairment in the placenta, analogous to the lesions characteristic of human preeclampsia (28).

Besides the down-regulation of IDO, the decrease in $\mathrm{CD} 4{ }^{+} \mathrm{CD} 25^{+} \mathrm{FoxP} 3^{+} \mathrm{T}$-cells is a potential factor in the pathogenesis of preeclampsia. Steinborn et al (7) and Sasaki et al (8) found a decreasing number of Treg cells present in preeclamptic women, and sugested that this change in Tregs might break the maternal tolerance to the fetus and result in preeclampsia. In this study, we detected the expression of FoxP3 mRNA by quantitative PCR, and revealed that its levels were markedly decreased in preeclamptic placentae.

We therefore hypothesized that the down-regulation of IDO expression and the decrease in the number of Tregs are closely related, and conducted further analyses to investigate whether the down-regulation of IDO affected the number of Tregs in preeclampsia. The preeclamptic samples were divided into two groups according to their IDO immunostaining score. The level of FoxP3 mRNA was lower in the samples with a low score, compared those with a high score. A positive correlation between IDO and FoxP3 mRNA levels was also found in the preeclamptic specimens. This suggests that IDO activity is related to the reduction in Treg cells, which participate in the pathogenesis of preeclampsia.

It has been suggested that IDO is a possible immunosuppressive effector mechanism of Tregs. Tregs can trigger high levels of functional IDO expression in mouse dendritic cells (DCs) in vitro (29). Conversely, it was recently revealed that IDO can encourage naive $\mathrm{CD}^{+}{ }^{+} \mathrm{T}$ cells to differentiate into Foxp $3^{+}$Tregs in vitro (30). A series of studies have provided evidence that IDO may play an important role in the proliferation and conversion of Tregs, as well as in the activation of preexisting resting Tregs. Besides DCs, IDO is expressed in endothelial cells and alters endothelial T-cell responses (31). In a study by Hautz et al (32), increasing expression of IDO and of FoxP3 was observed in the endothelium during rejection late after transplantation. The authors reported that characteristics of the cellular infiltrate indicated tolerogenic properties of a number of the cells, and thus a tendency of the alloimmune response towards self-limitation, during skin rejection after hand transplantation. Since, in placentae, most of the cells positive for IDO staining are endothelial cells, and since the down-regulation of IDO in endothelial cells is observable, it is reasonable to speculate that the down-regulation of IDO expression in endothelial cells may lead to a decrease in the number of Tregs, and thus play a role in the pathogenesis of preeclampsia.
In conclusion, we demonstrated that levels of IL-18, IDO and FoxP3 mRNA are decreased in preeclamptic placentae. The reduction of IDO mRNA is related to the level of FoxP3. Therefore, the down-regulation of IDO in preeclampsia may be partly influenced by IL-18 and result in the maladaptation of maternal tolerance and the pathogenesis of preeclampsia by directly reducing Treg cells. This is potentially a novel mechanism in the pathogenesis of preeclampsia, and warrants further investigation for the possible establishment of presymptomatic diagnosis or prophylaxis therapy.

\section{References}

1. Williams DJ and De Swiet M: The pathophysiology of preeclampsia. Intensive Care Med 23: 620-629, 1997.

2. Van Beek E and Peeters LL: Pathogenesis of preeclampsia: a comprehensive model. Obstet Gynecol Surv 53: 233-239, 1998.

3. Sibai B, Dekker G and Kupferminc M: Preeclampsia. Lancet 365: 785-799, 2005.

4. Redman CW and Sargent IL: Latest advances in understanding preeclampsia. Science 308: 1592-1594, 2005.

5. Nishizawa H, Hasegawa K, Suzuki M, et al: The etiological role of allogeneic fetal rejection in preeclampsia. Am J Reprod Immunol 58: 11-20, 2007.

6. Santoso DIS, Rogers P, Wallacea EM, Manuelpillaia U, Walkerb D and Subakir SB: Localization of indoleamine 2,3-dioxygenase and 4-hydroxynonenal in normal and preeclamptic placentae. Placenta 23: 373-379, 2002.

7. Steinborn A, Haensch GM, Mahnke K, et al: Distinct subsets of regulatory $\mathrm{T}$ cells during pregnancy: is the imbalance of these subsets involved in the pathogenesis of preeclampsia? Clin Immunol 129: 401-412, 2008.

8. Sasaki Y, Darmochwal-Kolarz D, Suzuki D, et al: Proportion of peripheral blood and decidual CD4(+) CD25(bright) regulatory T cells in Preeclampsia. Clin Exp Immunol. 149: 139-145, 2007.

9. Munn DH, Zhou M, Attwood JT, et al: Prevention of allogeneic fetal rejection by tryptophan catabolism. Science 281: 1191-1193, 1998.

10. Kudo Y, Boyd CA, Spyropoulou I, et al: Indoleamine 2,3-dioxygenase: distribution and function in the developing human placenta. J Reprod Immunol 61: 87-98, 2004.

11. Munn DH, Sharma MD, Hou D, et al: Expression of indoleamine 2,3-dioxygenase by plasmacytoid dendritic cells in tumordraining lymph nodes. J Clin Invest 114: 280-290, 2004.

12. Uyttenhove C, Pilotte L, Théate I, et al: Evidence for a tumoral immune resistance mechanism based on tryptophan degradation by indoleamine 2,3-dioxygenase. Nat Med 9: 1269-1274, 2003.

13. Lee JR, Dalton RR, Messina JL, et al: Pattern of recruitment of immunoregulatory antigen-presenting cells in malignant melanoma. Lab Invest 83: 1457-1466, 2003.

15. Nakanishi K, Yoshimoto T, Tsutsui H, Okamura H: Interleukin-18 regulates both Th1 and Th2 responses. Annu Rev Immunol 19: 423-474, 2001.

16. Huang X, Huang H, Dong M, Yao Q, Wang H: Serum and placental interleukin-18 are elevated in preeclampsia. J Reprod Immunol 65: 77-87, 2005.

17. Seol HJ, Lee ES, Jung SE, et al: Serum levels of YKL-40 and interleukin-18 and their relationship to disease severity in women with preeclampsia. J Reprod Immunol 79: 183-187, 2009.

18. Adams KM, Mandel LS, Guthrie KA, Atkinson MW: Interleukin-18 in the plasma of women with preeclampsia. Am J Obstet Gynecol 188: 1234-1237, 2003.

19. Roland L, Gagne A, Belanger MC, Boutet M, Julien P, Bilodeau JF: Plasma interleukin-18 (IL-18) levels are correlated with antioxidant vitamin coenzyme Q10 in preeclampsia. Acta Bestet Gynecol Scand 89: 360-366, 2010.

20. Liebau C, Baltzer AW, Schmidt S, et al: Interleukin-12 and interleukin-18 induce indoleamine 2,3-dioxygenase (IDO) activity in human osteosarcoma cell lines independently from interferon-gamma. Anticancer Res 22: 931-936, 2002.

21. Perry IJ and Beevers DG: The definition of pre-eclampsia. Br J Obstet Gynaecol 101: 587-591, 1994..

22. Kudo Y, Boyd CA, Sargent IL and Redman CW: Decreased tryptophan catabolism by placental indoleamine 2,3-dioxygenase in preeclampsia. Am J Obstet Gynecol 188: 719-726, 2003. 
23. Brewster JA, Orsi NM, Gopichandran N, McShane P, Ekbote UV, Walker JJ: Gestational effects on host inflammatory response in normal and pre-eclamptic pregnancies. Eur J Obstet Gynecol Reprod Biol 140: 21-26, 2008.

24. Ekelund CK, Vogel I, Skogstrand K, et al: Interleukin-18 and interleukin-12 in maternal serum and spontaneous preterm delivery. J Reprod Immunol 77: 179-185, 2008.

25. De la Maza LM and Peterson EM. Dependence of the in vitro antiproliferative activity of recombinant human gammainterferon on the concentration of tryptophan in culture media. Cancer Res 48: 346-350, 1988.

26. Bauer TM, Jiga LP, Chuang JJ, Randazzo M, Opelz G and Terness P. Studying the immunosuppressive role of indoleamine 2,3-dioxygenase: tryptophan metabolites suppress rat allogeneic T-cell responses in vitro and in vivo. Transpl Int 18: 95-100, 2005.

27. Mellor AL, Keskin DB, Johnson T, Chandler P and Munn DH Cells expressing indoleamine 2,3-dioxygenase inhibit $\mathrm{T}$ cell responses. J Immunol 168: 3771-3776, 2002.
28. Nishizawa H, Hasegawa K, Suzuki M, et al: Mouse model for allogeneic immune reaction against fetus recapitulates human Preeclampsia. J Obstet Gynaecol Res 34: 1-6, 2008.

29. Fallarino F, Grohmann U, Hwang KW, et al: Modulation of tryptophan catabolism by regulatory $\mathrm{T}$ cells. Nat Immunol 4: 1206-1212, 2003.

30. Fallarino F, Grohmann U, You S, et al: The combined effects of tryptophan starvation and tryptophan catabolites down-regulate $\mathrm{T}$ cell receptor zeta-chain and induce a regulatory phenotype in naive T cells. J Immunol 176: 6752-6761, 2006.

31. Mouratidis PX and George AJ. Transfection of indoleamine 2,3 dioxygenase in primary endothelial cells. Methods Mol Biol 616: 149-159, 2010.

32. Hautz T, Brandacher G, Zelger B, et al: Indoleamine 2,3-dioxygenase and foxp3 expression in skin rejection of human hand allografts. Transplant Proc 41: 509-512, 2009. 